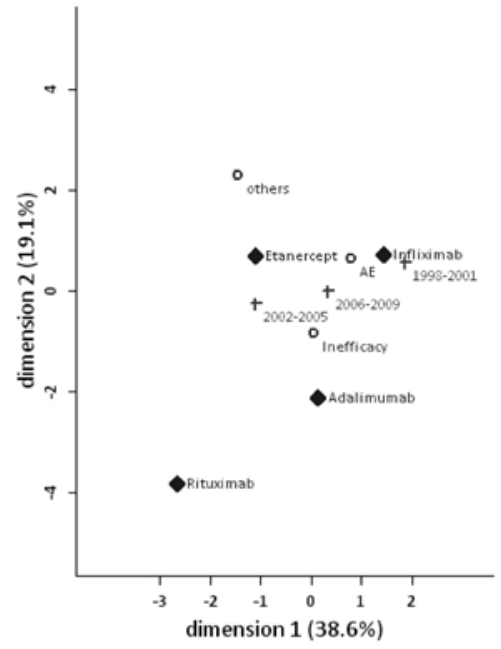

Abstract A10.24 Figure 1

Objective To visually assess the relationship between the reason for stopping the first biologic and the period of time when the biologic was started.

Methods All patients with rheumatoid arthritis undergoing treatment with a biologic are registered in the clinical database of a single centre with seven treating rheumatologists. The biologic used, as well as the reason to stop it is recorded, as well as all dates regarding the start and the last dose. We performed a multiple correspondence analysis with the Burt method of adjusted inertias and standard normalisation with the variables: first biologic, time period (1998-2001, 2002-2005, 2006-2009), and reason to stop (adverse event, inefficacy, others). Correspondence analysis provides a means of displaying or summarising a set of data with categorical variables in two-dimensional graphical form.

Results The total inertia of the exploratory analysis was 0.10 . The first two dimensions added to $57 \%$ of the inertia, so that all dimensions were accounted for. The major drives of the relationship between the three variables were "infliximab" and the time period "1998-2001" (\% inertia 0.146 and 0.177 , respectively). The distance between other categories of the three variables was not relevant enough to make any suggestions of an association. The figure shows the correspondence map of the relationship.

Conclusions If to any biologic at all, time has affected the decision to start infliximab, with more treatments started in the earlier times than in the later ones. The reason to stop any first biologic does not clearly relate to the time neither to the biologic itself in this single centre dataset.

\section{A10.25 SYNOVIAL TISSUE TRANSCRIPTOME AND SYNOVIAL FLUID PROTEOME HAVE STRONGER DISCRIMINATORY POWER THAN SERUM IN DISSECTING INFLAMMATION IN RA}

doi:10.1136/annrheumdis-2013-203224.25

'Biljana Smiljanovic, 'Bruno Stuhlmueller, 'Silvia Pade, 'Marina Backhaus, 'Gerd-R Burmester, ${ }^{2}$ Andreas Radbruch, ${ }^{2}$ Andreas Grützkau, 'Thomas Häupl. 'Department of Rheumatology and Clinical Immunology, Charité CCM, Charitéplatz 1, 10117 Berlin, Germany; ${ }^{2}$ German Rheumatism Research Center - Berlin (DRFZ-Berlin), Ein LeibnizInstitut, Charitéplatz 1, 10117 Berlin, Germany

Background and Objectives Rheumatoid arthritis (RA) is a chronic disease associated with polyarticular inflammation, cartilage and/or bone destruction. Current diagnosis of RA is based on clinical criteria and measurement of non-specific makers of inflammation in the blood. A main challenge in diagnosis of RA is to establish objective criteria able to provide a more detailed insight into joint pathobiology. In search for convenient biomarkers, geneexpression profiles from synovial tissues of RA and osteoarthritis $(\mathrm{OA})$ patients were generated. These profiles were used for selection of candidate biomarkers that were measured at the protein level both in synovial fluid and matched serum samples from RA and OA patients.

Materials and Methods Gene-expression profiles from synovial tissues of RA and OA patients were generated by Affymtetrix HGU133A arrays. The BioRetis database was used for array analyses and selection of RA candidate genes. ELISA and multiplex immunoassays were used for validation of the candidate markers at the protein level.

Results Transcriptome analyses of synovial tissues from RA and OA patients revealed more than 1000 differentially expressed genes. Genes involved in chemotaxis, inflammation, cell adhesion and activation were strongly up-regulated in patients with RA. In total, 20 genes out 1000 were used for validation at the protein level, including 1) cytokines: IL8, MIF, MIG, CXCL13, CCL18; 2) the shedded form of membrane molecules: sCD14, sCD163, sCD44, uPAR, sICAM1, sVCAM1, P- Selectin, E-Selectin; and 3) markers related to bone destruction and bone formation: NTxI and PINP. These molecules were measured both in SF and matched serum samples from RA and OA patients. Protein levels of all these markers were elevated in SF of RA patients, showing an obvious inflammatory profile that clearly discriminated RA from OA. Serum levels of these markers were elevated in RA patients, compared to OA and/or healthy donors (ND) as well. Among all serum markers CXCL13, sCD14, CCL18, PINP and NTxI disclosed the highest level of specificity and sensitivity. Nevertheless, the potential of serum markers in general to discriminate RA from OA and/or ND was far weaker compared to those in SF.

Conclusions Disease-specific profiles were evident in the joint where both synovial tissue transcripts and related SF proteins revealed a clear differences between $\mathrm{RA}$ and $\mathrm{OA}$. Despite blood is a favourable diagnostic material, the results from this study indicated that the capacity of blood to dilute and neutralise inflammation is robust enough to limit discrimination between RA and OA.

\section{A10.26 SYNOVIAL Wnt AND WISP1 EXPRESSION INDUCES EXPRESSION OF CARTILAGE-DEGRADING METALLOPROTEINASES IN THE SYNOVIUM}

doi:10.1136/annrheumdis-2013-203224.26

M van den Bosch, A Blom, A van Erp, F van de Loo, E Blaney Davidson, P van der Kraan, $\mathrm{P}$ van Lent, W van den Berg. Radboud University Nijmegen Medical Centre, NCMLS, Nijmegen, Netherlands

Background Many osteoarthritis (OA) patients show synovial involvement. We found strong upregulation of Wnts $2 b$ and 16 and the downstream protein WISP1 in knee joints in experimental OA. The role of the synovium in the induction of OA pathology under the influence of Wnt signalling is unclear. Here we investigated the potential of Wnt signalling to increase the expression of cartilagedegrading enzymes in the synovium.

Methods Pathway analysis of microarray data from the synovium of a collagenase-induced OA mouse model was done using DAVID software. In vivo synovial overexpression of genes from the canonical Wnt signalling pathway was achieved by intra-articular injection of adenoviral vectors. Joint pathology was assessed by histology. Gene expression was analysed by qPCR. Human OA synovial specimens were collected from joint replacement surgery and stimulated or used for outgrowth of fibroblasts.

Results Pathway analysis showed that the Wnt signalling pathway was enriched in the synovium during experimental OA. To determine the effects of Wnt signalling on synovial tissue, we stimulated human OA synovial specimen with Wnt3a or WISP1, which resulted in increased expression of MMP3, MMP9 and MMP13, 